\title{
Hand Geometry and Palmprint Classification System Based on Statistical Analysis
}

\author{
Sarah Jasim Mohammed \\ Mechanical Engineering Department, University of Technology, Baghdad-Iraq. \\ Corresponding Author: Sarah_jasimm@yahoo.com.
}

\begin{abstract}
Biometric system is considered of an important type of security systems nowadays, because it relays on the individual traits (physical or behavioral) non-participation between any two people that can't be lost on lifetime and can't be stolen. This paper, will present the individual classification system based on hand geometry and palm texture feature where it is one of the parts of the human body, which has an impressive set of information capable to distinguish and identify individuals. Utilize Principal Component Analysis PCA for palimprint texture feature extraction. The proposed system consists of three phases: image preprocessing, hand feature extraction and pattern classification. Utilize Principal Component Analysis PCA for palimprint texture feature extraction. The proposed system utilized complete hand image inside database consists of 600 pictures, include 100 people, each one has six images. Experimental results show that $98.3 \%$ is achieved and that illustrate the applicability of the system in the security's average of different environments.
\end{abstract}

[DOI: $10.22401 /$ JNUS.20.4.17]

Keyword: Biometric hand geometry, palm texture feature, Principal Component Analysis.

\section{Introduction}

Biometric system, defined as the science of distinguishing an individual, depend on physical/ behavioral traits. Physical biometrics include traits of an individual, such as fingerprint, face, iris pattern and hand geometry. Behavioral biometrics include traits of an individual, such as signatures, keystroke, and voice[1]. In the last few decades the hand geometry has attracted a lot of attention because:

- Hand geometry is robust and reliable, not exposed to the circumstances of the environment.

- They have stable shape (finger length and width) through human lifetime.

- The palm texture has many unique features capable to recognize an individual.

- The person collaboration with a hand scanner is characterized as fast and friend user.

- They needs small information storage 20 bytes - so putting away, seeking and comparing them could be possible immediately even on low hardware.

- Low-cast, it needs to palm scanner device [2].

There are many approaches of Biometric systems that use human hand, some of them based hand geometry, some of them based palm texture feature or based palm vein characteristics [3]. Hand geometry features include finger length, width, curvature, thickness, width palm and relative position of these features [4]. A palm texture feature which include the contents of the texture that has basic lines, minutiae, wrinkles, delta points etc. that found in a palm of the hand. Palm vein characteristics include map of the veins in the palm of the hand [5].

Hand geometry system has the longest application history of biometrics manner. David Sidlauskas patented and developed the hand geometry conception in 1985 and the premier commercial hand geometry recognition system the next year became available, he debates a 3D hand profile identification device utilized in hand geometry [6]. Sanchez Reillo et. al. designated system depend on finger attributes (heights, widths and deviations) used to form a feature vector, his method a chieved $96 \%$ success, using Gaussian Mixture Modeling on a database of 20 persons with 10 hand images for each [7]. Duta, et al. elicited the feature points lie beside the main line of palms and computes a score that fits between a set of associated features of the two hands [8]. Jain et. al. presented a handgeometry relied verification system to utilize it 
for a proto-type web security system [9]. Oden et. al. developed a system utilizing implicit polynomials, it achieve a verification rate of 99\% [10]. Han et. al. extract the information found in a palm print for individual identification using morphological and Sobel edge and utilized a neural-network classifier for verification[11]. Kumar et.al. proposed system relied on palm-print and hand geometry utilized a simple matching score method [12]. Dewi Y.L., Eries T. U. Implemented palm-recognition involved of block-based line detection which dealing with palm-print feature extraction process, chain code for the hand geometric feature extraction. $\mathrm{He}$ mixed features and recognized it using Dynamic Time Warping (DTW) method [13]. Nidhi S., Vipul S., Neelesh D. and Pragya M. Presented Hand geometry system include compute the lengths and widths of fingers and the width of a palm [14]. Dale M. P., Joshi M. A., Galiyawala H. J. proposed system relied on hand geometry and feature fusion transform, the proposed algorithm divides the palm-print image into four non-overlapping parts around center point and applied discrete transforms (DCT) and (DFT) transforms for each subimage, then used Euclidian distance for hand identification [15].

Texture feature analysis is the significant method that utilize in various image processing fields such as classification, segmentation and pattern recognition. Texture analysis is occurring in many methods, several of the most important is statistical methods [16]. In 1901 Karl Pearson proposed the Principal Component Analysis (PCA). It is also known as Karhunen Loeve. PCA is utilized for the reduction of higher dimensional datasets to the lower dimensions for data analysis and feature extraction. The original data is transformed into a much smaller dimensional feature space. Generally, this analysis included the calculation of eigenvalue decomposition and singular value decomposition for data covariance matrix and data matrix respectively after mean entering the data of each feature [17].

In this paper, the proposed algorithm utilizes hand geometry and palm texture feature to individual classification based on digital image processing that including hand preprocessing, feature extraction, and pattern classification.

\section{Methodology}

The proposed hand classification system consist of three main stages that are shown in the block diagram:

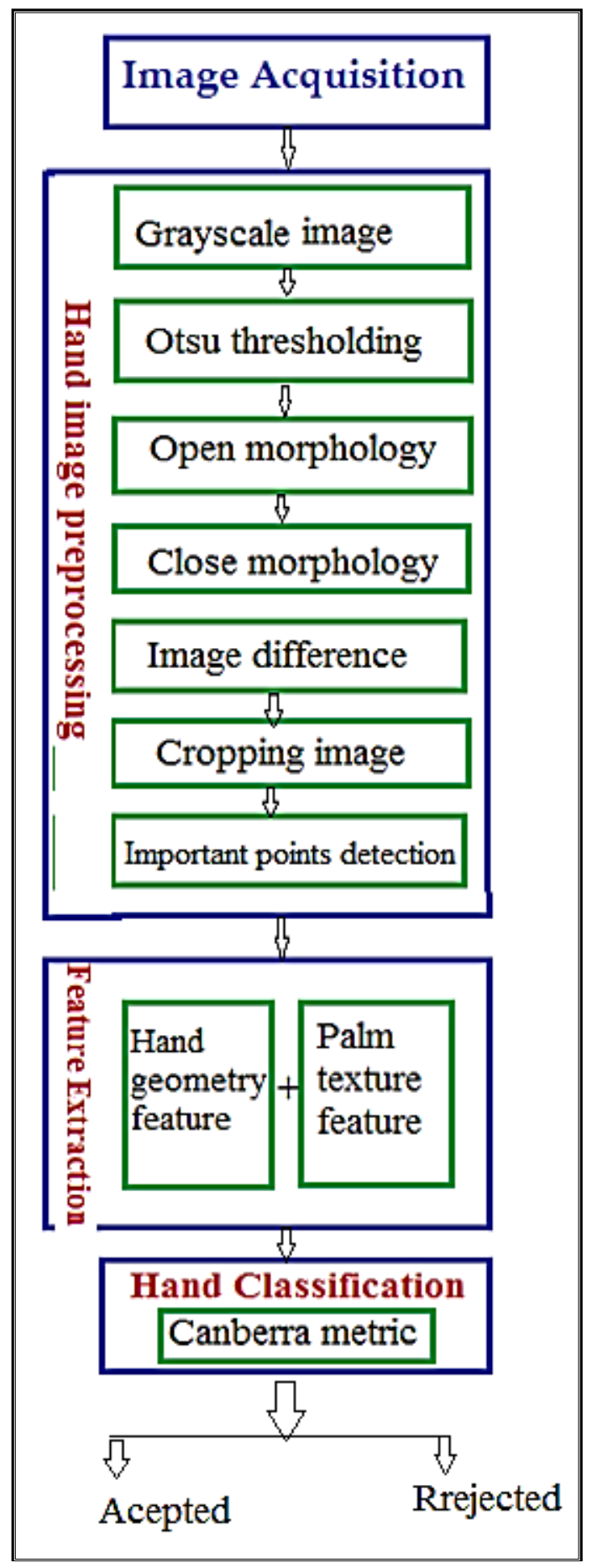

Fig.(1) : Block Diagram of the proposed system. 


\section{Hand image preprocessing}

The proposed algorithm of hand image processing is shown below:

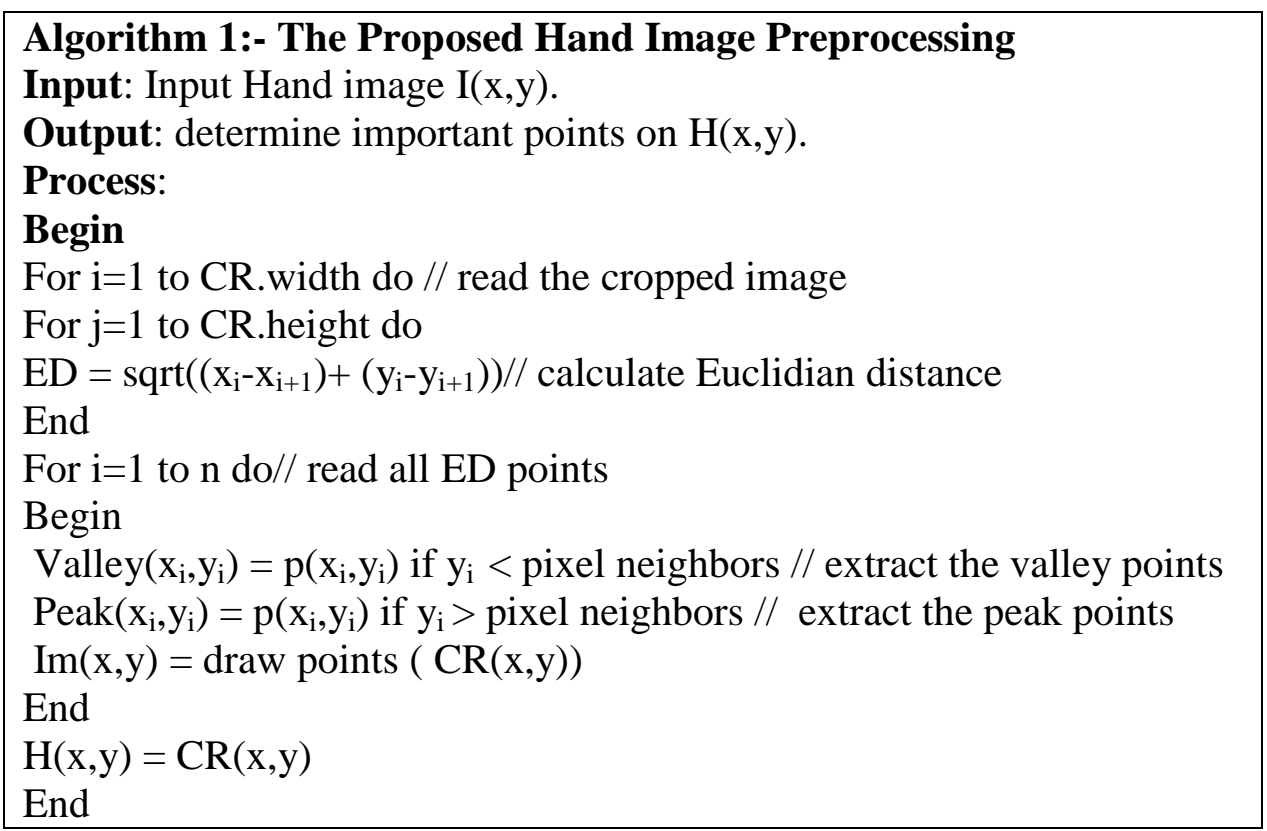

The proposed system reads the hand image captured by the sensor which be colored, then converting to grayscale. The grayscale image is binaries by using Otsu thresholding method. To extract hand boundary we used morphological operation (open and close) on binary images, on the resulting image applied image difference to obtain the hand contour clearances. After boundary extraction crop an image to include the shape of the hand only to reduce the space and process. The next step is detect the important points on the hand boundary such as reference point (the lower left-most pixel), peak and valley points by applied distance calculation on hand boundary pixels. Thus this stage end with fixed the important points on grayscale image which utilized in the feature extraction stage. Fig.(2) shows the steps of hand preprocessing.

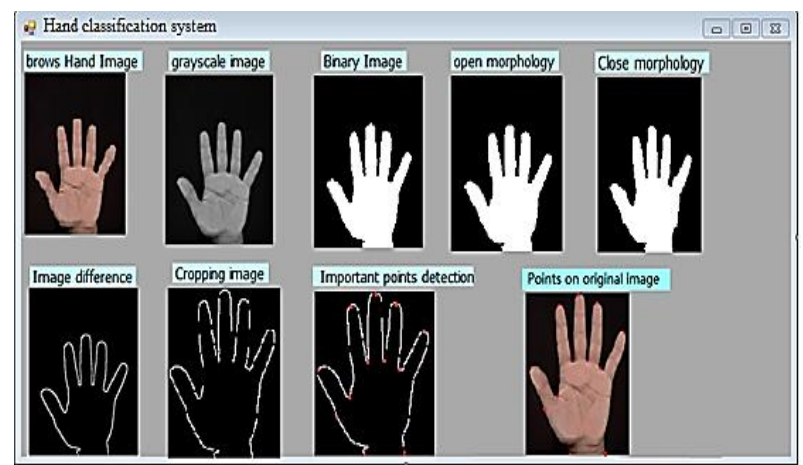

Fig.(2) : Hand image preprocessing.

\section{Feature Extraction}

The proposed system extracts the hand features based on hand geometry and palm texture feature. The proposed algorithm is shown below: 


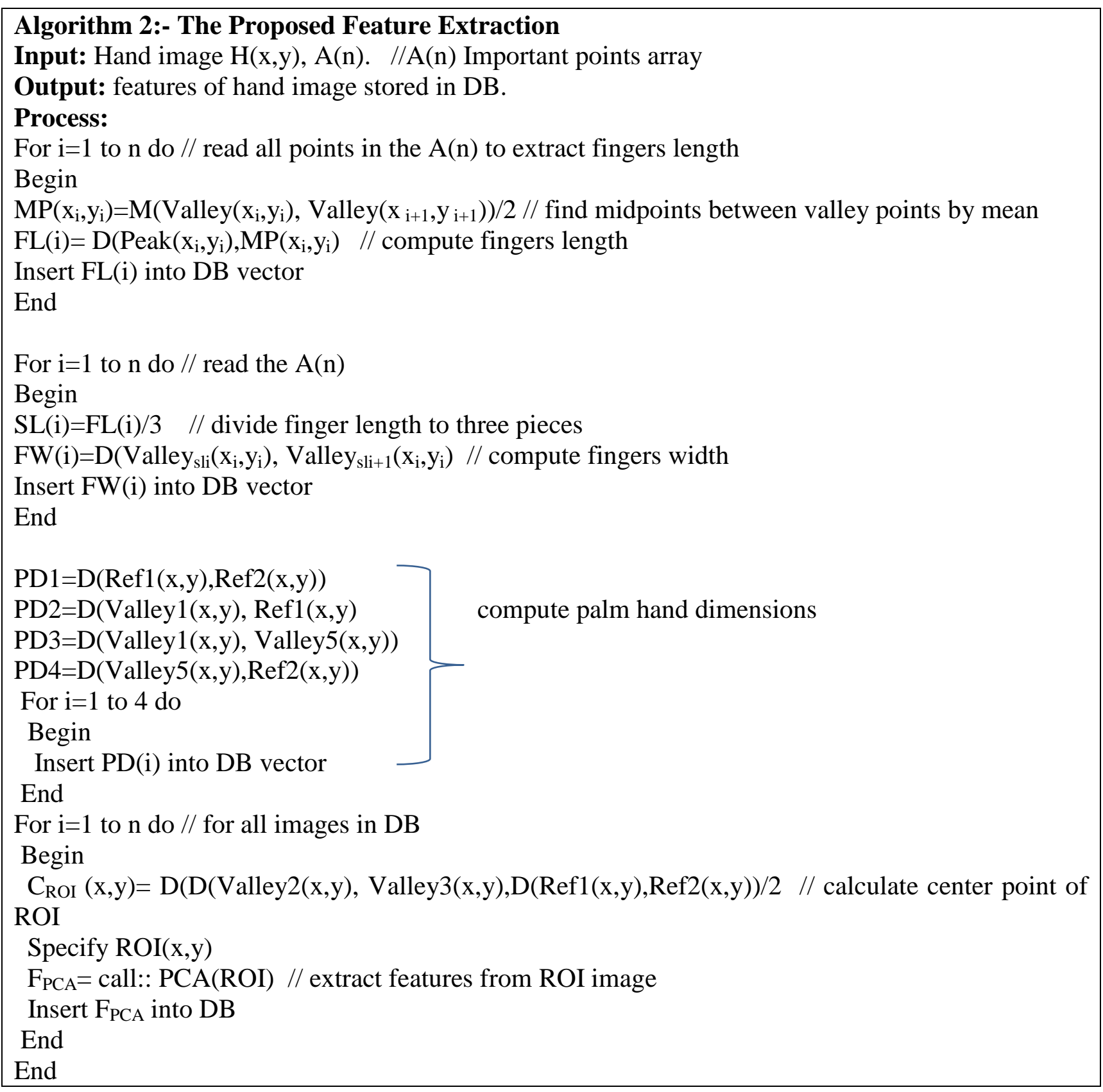

The first step is calculated hand geometry feature that includes (finger lengths, finger widths, palm dimensions) by utilized Euclidian distance between two pixels based on peak and valley points, reference point. The equation of Euclidian distance is defined as below[18]:

$E D=\sqrt{\left(x_{1}-x_{2}\right)^{2}+\left(y_{1}-y_{2}\right)^{2}}$

It calculates the length and width of the five fingers, each finger length is calculate the distance from the peak point to the midpoint between the valley points surrounded it. The finger width calculates in three positions, depending on the division of finger length to three pieces as shown in Fig.(3). The palm dimensions is calculated by finding the distance between valley points located on the hand borders and reference points. As a result we will get four features for each finger (1 length +3 width) and 4 features for hand palm. Thus, each hand are characterized by 24 geometry features from the other arranged in a feature vector which stored in a database. 


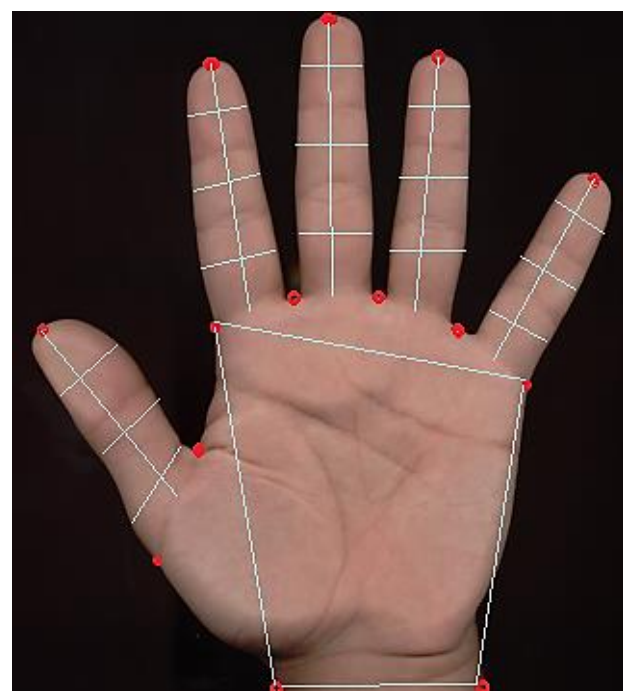

Fig.(3): Hand geometry feature.

The second step is extract palm texture features. The process starts by assigning the region that contains the largest number of features that are the middle of the hand palm. This area is determined by assigning the midpoint of the distance between the valley point of the middle finger and a point located midway between the reference points. Then, determine the center point of this distance, this point is the center an area call Region of Interest ROI and fixed equal distances from the right and left and top and bottom to determine the ROI as shown in Fig.(4).

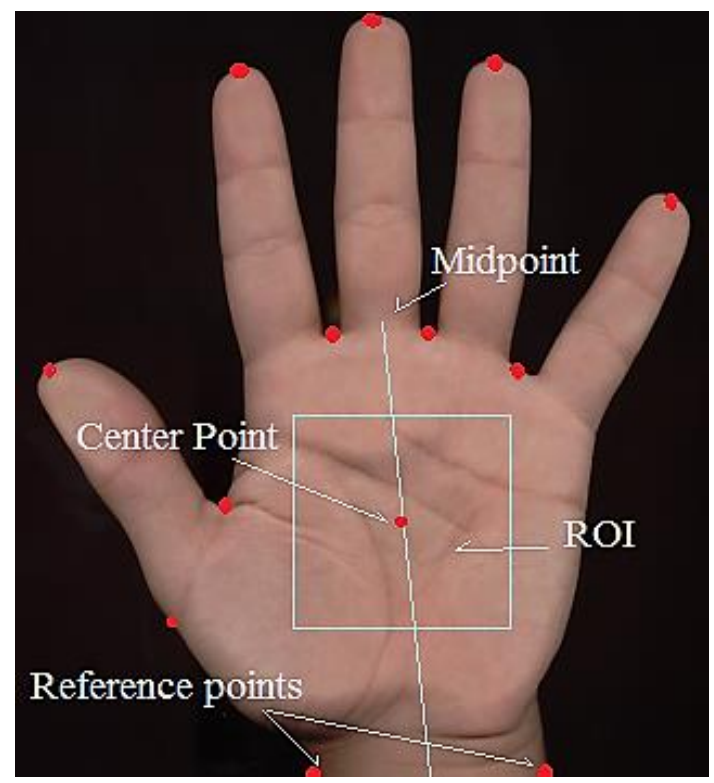

Fig.(4): ROI determination.

The proposed system extracted palm texture feature specified by ROI using Principal Component Analysis PCA, which enabling the system to distinguish the hands of the individual from others accurately. Each ROI image is transformed into a vector called eigenpalm by a sequence of related rows, in addition, the co-variance matrix is computed from training ROI images for the same individual that is shown below[17]:

- compute the mean $\mathrm{H}(\mathrm{x})$ of ROI image for every dimension of dataset

$$
H(x)=\frac{1}{N} \sum_{n=1}^{x} X_{n}
$$

Where $\mathrm{N}$ is number of ROI image

- Compute variance to obtain improved cooperative of vectors

$$
\bar{x}_{n}\left\{X_{n}-H(x)\right\}
$$

- Compute co-variance matrix

$$
C V=X^{T} * X
$$

- Compute eigenpalm space using eigenvectors and eigenvalues of co-variance matrix where $\mathrm{EV}=[\mathrm{ev} 1, \mathrm{ev} 2 \ldots . . \mathrm{evn}]$ signifies a matrix including eigenvectors of $\mathrm{CV}$ with dimension $\left(\mathrm{n}^{*} \mathrm{n}\right)$

$$
E P=E V * X^{T}
$$

- Compute feature vectors using eigenpalm

$$
F P=E P * X
$$

Where FP in $\left(n^{*} n\right)$ dimension, $n$ columns represent eigenpalm in a training set.

Now, features of eigenpalm are gained, can store as a set in a feature vector in addition to geometry features which stored in a database as show in Table (1) to use in classification stage. 
Sarah Jasim Mohammed 


\section{Hand Classification}

In the classification phase, the similarity between the test image sample and all trained images stored in the database is computed and the result used for classification. In this paper, Canberra metric is used to find similarity degree between two vector $(p, q)$, where $p$ is test image feature vector and $\mathrm{q}$ is image stored feature vector, it is defined as below [19]:

$$
d(p, q)=\sum_{i=1}^{n} \frac{\left|p_{i}-q_{i}\right|}{\left|p_{i}\right|+\left|q_{i}\right|}
$$

Classification decision of the test image sample depends on the degree of similarity where the test image sample is similar to the image stored in the database if the distance is the smallest value.

\section{Results and Discussion}

Experimental calculation of classification system is carried on the color hand images collected in hand database consist of 100 person each one has six images three left and three right. The hand image with resolution 382*526. The proposed system has implemented using $\mathrm{C \#}$ language run over Microsoft windows 10. The system includes two phases, training phase and the classification phase. In the training phase, train all hand images stored in a database through passing into system stage respectively, for transforming to vectors included hand geometry feature and palm-print texture feature to be ready to the classification stage, where hand image sample classify within database content.

The integration process of hand geometry feature and palm-print texture feature was appear increase the system reliability from if the proposed system applied based on hand geometry feature separately from palm-print texture feature, where result proves that the system classification accuracy is $98.3 \%$ and the average runtime required its estimated about (168 sec) as shown in Table (2). The accuracy of the system can be compute using the following accuracy equation[2]:

$$
\text { Accuracy }=\frac{[T P+F N]}{[T P+T N+F P+F P]}
$$

Table (2)

The proposed system accuracy.

\begin{tabular}{|l||c||c|}
\hline $\begin{array}{c}\text { Feature extraction } \\
\text { type in the proposed } \\
\text { algorithm }\end{array}$ & Accuracy & $\begin{array}{c}\text { Running } \\
\text { time }\end{array}$ \\
\hline \hline Hand geometry feature & $81 \%$ & $103 \mathrm{sec}$ \\
\hline $\begin{array}{l}\text { Palm-print texture } \\
\text { feature }\end{array}$ & $93.4 \%$ & $141 \mathrm{sec}$ \\
\hline $\begin{array}{l}\text { Hand geometry feature } \\
\text { and Palm-print texture } \\
\text { feature }\end{array}$ & $98.3 \%$ & $168 \mathrm{sec}$ \\
\hline
\end{tabular}

The comparison results of proposed system with others are given in Table (3).

Table (3)

The comparison system result with other related works.

\begin{tabular}{|l|l||c|c||}
\hline \multicolumn{1}{|c|}{ Method } & \multicolumn{1}{|c||}{ Procedure } & Number of people & Accuracy \\
\hline \hline Sanchez Reillo et. al., [7]. & $\begin{array}{l}\text { finger attributes (heights, widths } \\
\text { and deviations and using } \\
\text { Gaussian Mixture Modeling }\end{array}$ & 20 & $96 \%$ \\
\hline Dewi Y. L., Eries T. U.[13] & $\begin{array}{l}\text { combination of palmprint and } \\
\text { hand geometry using (DTW) }\end{array}$ & 50 & $89 \%$ \\
\hline $\begin{array}{l}\text { Nidhi S., Vipul S., Neelesh } \\
\text { D., Pragya M.[14] }\end{array}$ & Hand geometry features & 96 & $\% 97.44$ \\
\hline $\begin{array}{l}\text { Dale M. P., Joshi M. A., } \\
\text { Galiyawala H. J.[15] }\end{array}$ & $\begin{array}{l}\text { Single Sensor Hand Geometry } \\
\text { and Palm Texture Fusion }\end{array}$ & 100 & $99.5 \%$ \\
\hline \hline The proposed system & $\begin{array}{l}\text { Hand geometry feature and } \\
\text { palmprint classification based on } \\
\text { statistical analysis }\end{array}$ & 100 & $98.3 \%$ \\
\hline
\end{tabular}


The results show that the proposed methods gives faster performance when using of PCA because reduce the data dimension and extract important feature so its efficient method for feature extraction.

\section{Conclusions}

In this paper, hand geometry and palmprint classification system is proposed, which is relied on statistical analysis. The hand geometry features approach not enough to dependent method for individual classification, but when combine it with palmprint texture feature extraction specially using an accurate statistical analysis method like PCA, which is compress used data and extract small size template improved the classification rate. Using ROI in the center of palimprint is efficient idea because fixed on a small region include massive texture features resulting reduced process and increase feature accuracy.

\section{References}

[1]. Franjie El Khoury, "Iris Biometric Model for Secured Network Access", CRC Press/Taylor \& Francis Group, LLC, 2013.

[2]. Charles A. Shoniregun, Stephen Crosier, "Securing Biometrics Applications", Springer Science+Business Media, LLC, 2008.

[3]. N. Miura, A. Nagasaka, T. Miyatake, "Extraction of Finger-Vein Patterns Using Maximum Curvature Points in Image Profiles," Conf. on Machine Vision Applications, pp. 347-350, Japan, 2005.

[4]. Erdem Yörük, Ender Konuko glu, Bülent Sankur, Jérôme Darbon," Shape-Based Hand Recognition", IEEE transactions on image processing, VOL. 15, NO. 7, JULY 2006.

[5]. Kong, D. Zhang and G. Lu, "A study of identical twins palmprint for personal verification", Pattern Recognition, vol. 39, no. 11, pp. 2149-2156, 2006.

[6]. D.P. Sidlauskas, "3D hand-profile identification apparatus", US Patent, No.4736203, 1988.

[7]. R. Sanchez Reillo, "Hand Geometry Pattern Recognition through Gaussian Mixture Modeling," $15^{\text {th }}$, International Conference on Pattern Recognition, Vol. 2, pp. 937-940, September 2000.
[8]. N. Duta, A.K. Jain, K.V. Mardia, "Matching of Palmprints", Pattern Recognition Letters, pp. 477-485, 2002.

[9]. A. K. Jain and N. Duta, "Deformable Matching of Hand Shapes for Verfication," IEEE International Conference on Image Processing, pp. 857- 861, Oct.1999.

[10]. C. Öden, A. Erçil, B. Büke, "Combining implicit polynomials and geometric features for hand recognition," Pattern Recognit. Lett., Vol. 24, pp. 2145-2152, 2003.

[11]. C. Han, K C. Fan, H.L. Cheng, C. L. Lin, "Personal authentication using palmprint features," Pattern Recognition, Vol. 36, pp. 371-381, 2003.

[12]. Y. A. Kumar, A. K. Jain, "Personal verification using palm-print and handgeometry biometric", $4^{\text {th }}$ Int. Conf. Audio Video-Based Biometric Person Authentication, U.K., Jun. 9-11, pp.668678, 2003.

[13]. L. Dewi, U. Eries, "The combination of palm print and hand geometry for biometrics palm recognition" IJVIPNSIJENS, Vol: 12, No: 01.

[14]. S. Nidhi, S. Vipul, D. Neelesh, M. Pragya, "HAND GEOMETRY: A New Method for Biometric-Recognition", IJSCE, Vol. 2, Iss. 6, Jan. 2013.

[15]. P. Dale, A. Joshi, J. Galiyawala, "A Single Sensor Hand Geometry and Palm Texture Fusion for Person Identification", International Journal of Computer Applications, Vol. 42, No. 7, Mar. 2012.

[16]. P. Brodatz, "Textures: A Photographic Album for Artists and Designers". Reinhold, New York, 1986.

[17]. I. T. Jolliffe, "Principal Component Analysis", $2^{\text {nd }}$ ed, New York: SpringerVerlag, 2002.

[18]. R.W. Hamming, Error detecting and error correcting codes. Bell System Technical Journal, Vol. 26, No. 2, p.147160, 1950.

[19]. S.M. Emran, Ye N., "Robustness of Canberra metric in computer intrusion detection". Proceedings of the 2001 IEEE, Workshop on Information Assurance and Security, United States Military Academy, West Point, New York, 5-6 June, 2001. 